\title{
Is alcohol required for effective pancreatic cyst ablation? The prospective randomized CHARM trial pilot study
}

Authors

Institutions
Matthew T. Moyer ${ }^{1}$, Charles E. Dye ${ }^{1}$, Setareh Sharzehi ${ }^{1}$, Brooke Ancrile ${ }^{1}$, Abraham Mathew ${ }^{1}$, Thomas J. McGarrity ${ }^{1}$, Niraj Gusani ${ }^{2}$, Nelson Yee ${ }^{3}$, Joyce Wong ${ }^{2}$, John Levenick', Brandy Dougherty-Hamod', Bradley Mathers ${ }^{4}$

Institutions are listed at the end of article. submitted

3. November 2015 accepted after revision 25. February 2016

\section{Bibliography}

DOI http://dx.doi.org/ 10.1055/s-0042-105431 Published online: 10.5.2016 Endoscopy International Open 2016; 04: E603-E607 (c) Georg Thieme Verlag KG Stuttgart · New York E-ISSN 2196-9736

\section{Corresponding author} Matthew T. Moyer, MD Division of Gastroenterology and Hepatology

Penn State Hershey Medical Center

500 University Drive, Hershey, PA 17036

Fax: +1-717-531-4870

mmoyer@hmc.psu.edu
Background and study aims: In this study, we aim to determine the safety and feasibility of an alcohol-free approach to pancreatic cyst ablation using a chemotherapeutic ablation cocktail.

Patients and methods: In this prospective, randomized, double-blinded pilot study, 10 patients with known mucinous type pancreatic cysts underwent endoscopic ultrasound (EUS)-guided fine needle aspiration and then lavage with either $80 \%$ ethanol or normal saline. Both groups were then treated with a cocktail of paclitaxel and gemcitabine. Primary outcomes were reduction in cyst volume and rates of complications.

Results: At 6 months, patients randomized to the alcohol arm had an $89 \%$ average volume reduc-

\section{Introduction \\ $\nabla$}

Approximately 46,000 people will develop pancreatic cancer this year in the United States, and almost all are expected to die from the disease, representing the fourth leading cause of cancer associated mortality [1]. While approximately $90 \%$ of pancreatic cancers are ductal adenocarcinomas, a significant portion of pancreatic cancer develops from mucinous type pancreatic cysts. Pancreatic cystic lesions are increasingly encountered and typically discovered incidentally on cross-sectional imaging, occurring in approximately $2 \%$ of all American adults with a $37 \%$ prevalence in individuals older age 80 [2].

Discovering a pancreatic cyst presents a new set of difficulties, with options including either indefinite radiographic surveillance with magnetic resonance imaging (MRI) or computed tomography or surgical resection, both of which have significant limitations. Surveillance carries significant economic and possible psychological burdens while waiting for a possible malignancy to develop, as well as radiation exposure with CT. On the other hand, surgical resection possesses a significant risk of adverse events (AEs) $(20 \%-40 \%)$ and tion, with a $91 \%$ reduction noted in the alcoholfree arm. Complete ablation was achieved in $67 \%$ of patients in the alcohol-free arm at both 6 and 12 months, whereas the alcohol group recorded complete ablation rates of $50 \%$ and $75 \%$ at 6 and 12 months, respectively. One patient in the alcohol arm developed acute pancreatitis (20\%) with no adverse events in the alcohol-free arm.

Conclusions: This study revealed similar ablation rates between the alcohol ablation group and the alcohol-free arm and demonstrates the safety and feasibility of an alcohol-free ablation protocol. This pilot study suggests that alcohol may not be required for effective cyst ablation.

mortality $(1 \%-2 \%)[3-5]$. This clinical dilemma delineates the pressing need to develop effective, but more minimally invasive, approaches for the elimination of these premalignant pancreatic cysts, and among these, endoscopic ultrasound guided fine needle infusion (EUS-FNI) has emerged as an innovative and promising approach [6]. EUS-FNI was first well demonstrated by the EPIC trial, a prospective, randomized trial which demonstrated a $33 \%$ rate of complete cyst ablation following EUS-guided cyst lavage with $80 \%$ ethanol when followed over 6 months with a major AE rate limited to pancreatitis $(4 \%-5 \%)$ [7]. To increase the ablative effect, Oh et al added the innovative step of infusing and leaving paclitaxel in the cyst after ethanol lavage [8]. The addition of paclitaxel, a chemotherapeutic agent which arrests cellular microtubule assembly thus interfering with $G_{2}$ mitotic-phase cell replication, was shown in 3 separate trials to raise complete ablation rates to $60 \%-79 \%[6,8]$. Importantly, the principle $\mathrm{AE}$ in trials to date has been acute pancreatitis, with occurrence rates of $2 \%$ to as high as $10 \%[6,9]$ which was typically severe enough to result in hospitalization and has led some critics to question whether this risk profile is an issue 
[10]. Importantly, in previous trials, the mechanism of pancreatitis was felt to be secondary to extravasation of alcohol $[7,8]$.

As demonstrated by the 3 Oh trials, a dramatic increase in efficacy was demonstrated with the addition of the chemotherapeutic agent paclitaxel to the ablation process, and the reasons for this increase are unknown and have been speculated to be possibly a synergistic effect between alcohol and paclitaxel at the level of the cyst wall $[6,8]$. However, with the marked increase in ablation efficacy with the addition of paclitaxel to cyst ablation, the importance of alcohol in the ablation process is unclear. Therefore, this trial hypothesizes that alcohol is not required for effective cyst ablation when an ablation cocktail specifically tailored to pancreatic neoplasia is used, and that the removal of alcohol would directly improve the already favorable safety profile of this technique.

This study has 3 aims. We hypothesize that:

1. Alcohol is not required for effective pancreatic cyst ablation.

2. The removal of alcohol will decrease AE rates associated with pancreatic cyst ablation.

3. The efficacy of neoplastic pancreatic cyst ablation can be improved when a chemotherapeutic ablation cocktail specifically designed for pancreatic neoplasia is used.

The rationale behind the second aim comes from the spectrum of oncology practice where multi-agent formulations have become the standard of care in a variety of increasingly effective chemotherapy treatment protocols (FOLFOX-R, CHOP-R, etc.) by targeting multiple cellular mechanisms and receptor pathways. To design a more effective pancreatic cyst ablation cocktail, we chose paclitaxel as the first agent because of its efficacy previously shown in the Oh trials. Because paclitaxel $(6 \mathrm{mg} / \mathrm{mL})$ is highly viscous, reducing the viscosity with normal saline is required for needle infusion. In this trial, we planned to replace the normal saline with gemcitabine (2',2'-difluorodeoxy-cytidine), a cytotoxic agent with proven efficacy against a number of solid tumors that has been the backbone of pancreatic cancer chemotherapy for over 30 years. Gemcitabine is a prodrug which after intracellular phosphorylation exerts its cytotoxic effects through active intracellular metabolites arresting deoxyribonucleotide synthesis, leading to termination of DNA synthesis and cell death $[11,12]$. When reconstituted with normal saline, its viscosity is extremely low, making it an ideal second agent to dilute paclitaxel and add a second cellular treatment pathway. In addition, recent phase 2 trials have shown the combination of paclitaxel and gemcitabine to improve overall progression-free survival, as well as response rates, in pancreatic cancer when compared to other standard of care chemotherapy treatments [13].

\section{Patients and methods \\ $\nabla$}

\section{Preclinical drug evaluation}

As per FDA request, initial bench top testing was carried out demonstrating that combining paclitaxel and gemcitabine in a syringe did not change the color or viscosity or form a visible precipitate over 24 hours, and that $2 \mathrm{~mL}$ of the admixture could be injected through a 22-gauge needle in under 2 minutes using a high-pressure gun. In addition, high performance liquid chromatography (HPLC) compatibility testing of the 2 separate agents ( $3 \mathrm{mg} / \mathrm{mL}$ paclitaxel and $19 \mathrm{mg} / \mathrm{mL}$ gemcitabine) first separately and then mixed in the same syringe demonstrated stable drug integrity for 24 hours (0 Fig. 1).

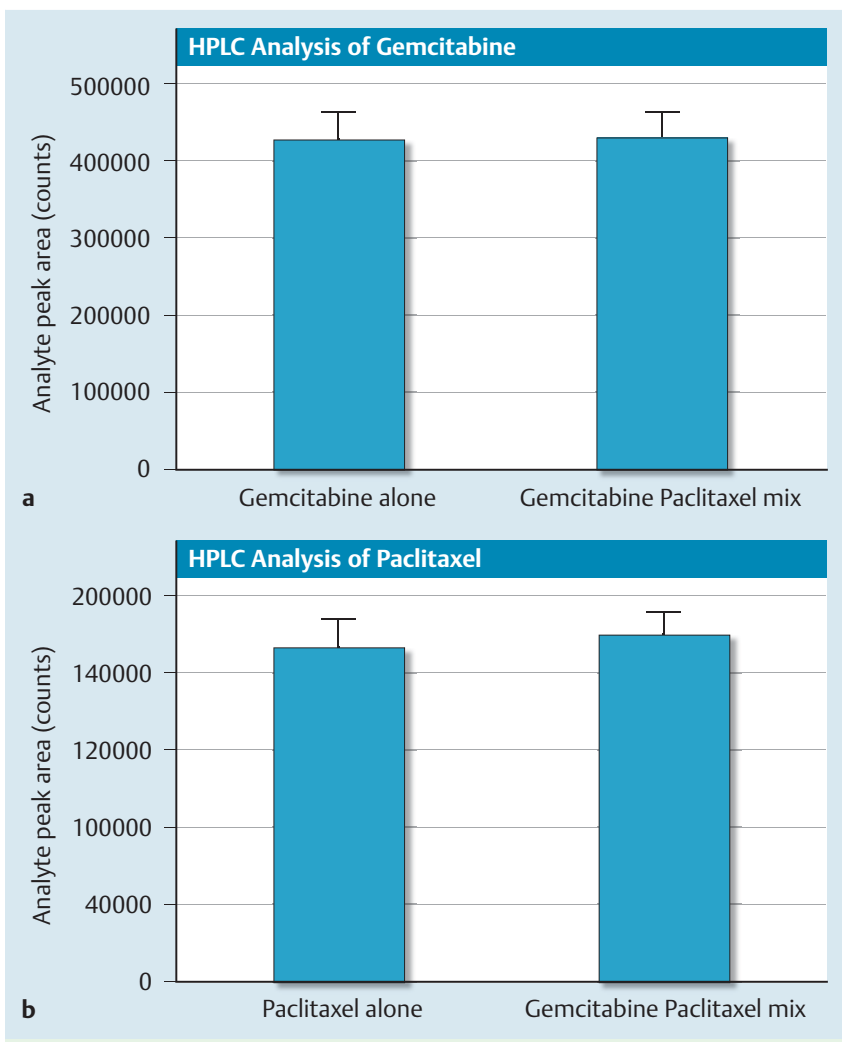

Fig. 1 In use compatibility and stability testing of gemcitabine/paclitaxel, as measured by HPLC analysis. Gemcitabine (Gemzar, Eli Lilly, $38 \mathrm{mg} / \mathrm{mL}$ ) and paclitaxel (Hospira, $6 \mathrm{mg} / \mathrm{mL}$ ) were mixed 1:1 in a syringe and stored at room temperature for 24 hours. Analyte peak area was measured to indicate the amount of gemcitabine (a) and paclitaxel (b) in the original formulations compared to the 1:1 mixture.

\section{Study design}

This investigator-initiated, prospective, randomized, doubleblinded, single-center trial was approved by the Institutional Review Board and registered as a clinical trial (ClinicalTrials.gov, NCT01475331) and carries the appropriate Food and Drug Administration (FDA) IND approval (\#111159). Eligible patients included those referred to this institution's gastroenterology or surgical oncology programs with a pancreatic cyst(s) previously identified by cross-sectional imaging or endoscopic ultrasound. The principal investigator subsequently reviewed the medical records and imaging to assess eligibility. Candidates were then seen in clinic for evaluation, consultation and consenting. If not already performed, patients were offered a consultation with surgical oncology to further review their surgical options.

Inclusion criteria included: patients older than age 18 who were able to give voluntary, written, informed consent and safely undergo endoscopy with deep sedation, a known pancreatic cystic lesion of 1 to $5 \mathrm{~cm}$ with fewer than 5 compartments, no intimate communication with the main pancreatic duct, or evidence of pancreatitis.

Patients were excluded for: known or suspected pancreatic cancer, pathologic lymphadenopathy, irreversible coagulopathy, white blood cell counts greater than 14,000 or less than 2,000 , hematocrit less than 30, platelets less than 30,000, CA19-9 greater than $40 \mathrm{U} / \mathrm{mL}$, lipase greater than 3 times the upper limit of normal, or positive beta-hCG. Patients were also excluded if the clinical picture and cross-sectional imaging were consistent 
with a benign pancreatic cyst (pancreatic pseudocyst or serous cystadenoma).

Randomization occurred in a predetermined 1:1 ratio, blinded to the endoscopists and patients, with study drugs managed by the Investigational Drug Pharmacy. This pilot study of 10 randomized patients was designed to demonstrate the safety and feasibility of an alcohol-free pancreatic cyst ablation protocol.

\section{Interventional protocol}

All EUS procedures were performed at the Penn State Hershey Medical Center by 2 experienced endosonographers (MTM, CED), each of whom had performed more than 2,000 EUS procedures prior to this study. Either deep propofol or general anesthesia was used for all patients with a complete pancreatic examination performed using a curvilinear array echoendoscope (GFUCT180; Olympus America, Center Valley, PA). The cystic lesion was identified and characterized with regard to location, diameter, number of septations, and high-risk features per 2012 consensus criteria [14]. Translumenal cyst aspiration (EUS-FNA) was then performed and if at least $1 \mathrm{~mL}$ of cyst fluid was retrieved, CEA and amylase levels, as well as cytology samples were obtained. A 22-gauge (Echotip; Cook Medical, Bloomington, IN) and 19gauge FNA needle (Expect Needle; Boston Scientific, Marlborough, MA) were utilized for cysts measuring 1.5 to $2.5 \mathrm{~cm}$ and 2.6 to $5.0 \mathrm{~cm}$, respectively. After initial aspiration, if the cyst was noted to immediately accumulate fluid, thereby suggesting pancreatic duct communication, the procedure was aborted. However, this did not occur at any point during the study. Then, with the needle tip in the exact center of the cyst, lavage was performed for 3 to 5 minutes using an unlabeled 10-mL syringe of the study agent with subsequent infusion of the paclitaxel-gemcitabine cocktail using a 30-cc syringe custom fitted to a highpressure gun (Alliance II; Boston Scientific, Natick, Mass) to allow timely infusion of the cocktail. Patients randomized to the control group underwent cyst lavage with $80 \%$ ethanol, after which time the alcohol was nearly completely aspirated, followed by the infusion of the chemotherapy cocktail of $3 \mathrm{mg} / \mathrm{mL}$ of paclitaxel (Taxol; Hospira, Lake Forest, IL) and $19 \mathrm{mg} / \mathrm{mL}$ of gemcitabine (Gemzar; Eli Lilly, Indianapolis, IN), infusing an amount equal to the original amount aspirated to reestablish the original cyst size and volume. In the experimental group, cysts were aspirated and lavaged in the same manner using normal saline, followed by infusion of the chemotherapeutic cocktail of $3 \mathrm{mg} / \mathrm{mL}$ paclitaxel and $19 \mathrm{mg} / \mathrm{mL}$ gemcitabine. A maximum volume of $8 \mathrm{~mL}$ of the chemotherapeutic cocktail could be administered, as per FDA stipulation.

\section{Post-treatment follow-up}

Post EUS-FNI care was identical to that for any EUS-FNA procedure; however, patients were monitored for 2 hours post-procedure with a subsequent follow-up telephone interview at 72 hours. As per FDA request, all study patients had a comprehensive set of labs at 2 weeks' time. To measure response to ablation treatment, an MRI scan (or CT if MRI scan contraindicated) was obtained at 3, 6, and 12 months for all patients, or as determined necessary on a case-by-case basis to evaluate any possible adverse event ( $\bullet$ Fig. 2). Patients could elect to undergo evaluation for surgical resection at any time, regardless of response.
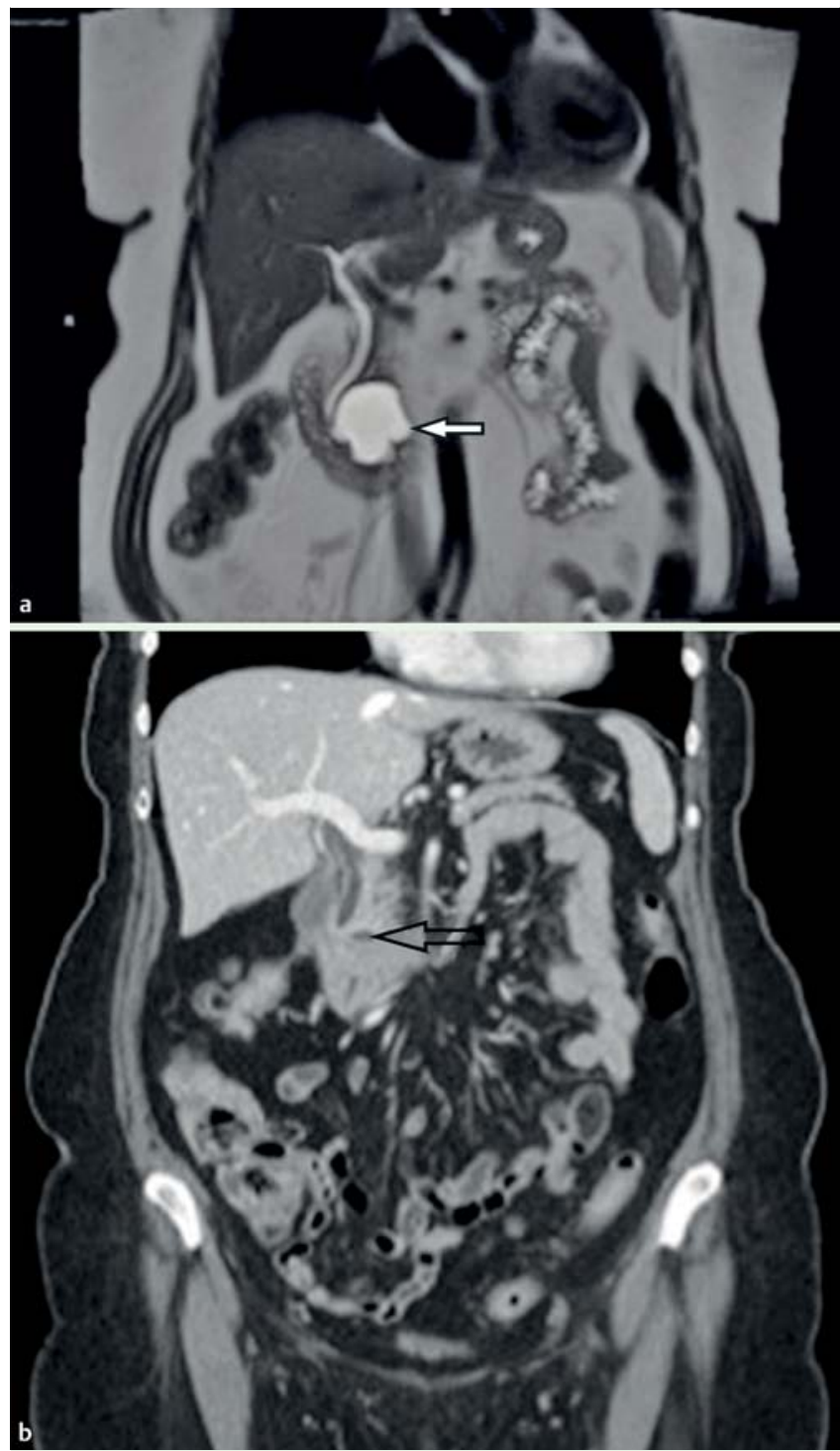

Fig. 2 a MRI image showing a 76-year-old female with a 4.2-cm mucinous (premalignant) type pancreatic cyst (white arrow) prior to EUS-guided chemoablation. b CT image showing a 4-mm residual defect at 1 year (black arrow).

\section{Study definitions}

Pancreatic cyst type was characterized based on the generally accepted schema, using clinical, radiographic, cytological, and chemical analysis with CEA and amylase levels [15]. Mucinous cystic neoplasms (MCN) were classified as lesions with CEA greater than $200 \mathrm{ng} / \mathrm{mL}$ and amylase less than $800 \mathrm{U} / \mathrm{L}$ while intraductal papillary mucinous neoplasms (IPMN) were classified as lesions with CEA greater than $200 \mathrm{ng} / \mathrm{mL}$ and amylase greater than 800 $\mathrm{U} / \mathrm{L}$. Cyst size was calculated by measuring $\mathrm{x}$ and $\mathrm{y}$ diameters and calculating cyst volume using the formula $43 \times \pi \times \mathrm{r}^{3}$ where $\mathrm{r}$ is the average of the cyst radius as measured on initial 3-, 6-, and 12-month MRI or CT. Response was defined according to the same volume percentage reductions as described in previous trials where: complete response $=\geq 95 \%$ reduction of cyst volume, partial response $=95 \%$ to $75 \%$ reduction and a non-response $=$ $<75 \%$ reduction in volume. [8] The overall ablation rates in both arms were compared to historical controls to assess the efficacy of the chemotherapeutic cocktail [6-8]. 
Table 1 Patient demographics and cyst characteristics $(n=10)$.

\begin{tabular}{|c|c|c|c|c|c|c|c|}
\hline & Age & Sex & Cyst Size (cm) & Cyst Type & Cyst Location & CEA Level (ng/ml) & Amylase Level (U/L) \\
\hline Pt. 1 & 82 & M & $2.2 \times 1.7$ & MCN & Body & 341.3 & $<30$ \\
\hline Pt. 2 & 69 & $\mathrm{~F}$ & $3 \times 1.8$ & MCN & Body & 1090.3 & $<30$ \\
\hline Pt. 3 & 73 & $\mathrm{~F}$ & 3.2 & MCN & Body & $>15000$ & $<30$ \\
\hline Pt. 4 & 47 & $\mathrm{~F}$ & 3.8 & MCN & Head & 897.4 & 104 \\
\hline Pt. 5 & 73 & $\mathrm{~F}$ & 3.7 & MCN & Body & 1001.9 & $<30$ \\
\hline Pt. 6 & 82 & $\mathrm{~F}$ & $4.2 \times 3.3$ & IPMN & Head & 18.8 & 5184 \\
\hline Pt.7 & 68 & $\mathrm{~F}$ & 2.4 & IPMN & Tail & 203 & 12000 \\
\hline Pt. 8 & 61 & $\mathrm{~F}$ & 2.3 & MCN & Head & 1085.8 & 295 \\
\hline Pt.9 & 81 & M & 2.2 & Indeterminate & Head & 109.7 & 300 \\
\hline Pt. 10 & 80 & $\mathrm{~F}$ & 2.1 & $\mathrm{MCN}$ & Body & 626 & 360 \\
\hline
\end{tabular}

Table 2 Results at 6 and 12 months post-procedure.

\begin{tabular}{|lllll}
\hline & $\begin{array}{l}\text { \% reduction in cyst size } \\
\text { after } \mathbf{6} \text { months }\end{array}$ & $\begin{array}{l}\text { \% reduction in cyst size } \\
\text { after } \mathbf{1 2} \text { months }\end{array}$ & $\begin{array}{l}\text { Complete response } \\
\text { after } \mathbf{6} \text { months }\end{array}$ & $\begin{array}{l}\text { Complete response } \\
\text { after } \mathbf{1 2} \text { months }\end{array}$ \\
\hline Alcohol arm & $89 \%$ & $94 \%$ & $2 / 4(50 \%)$ & $3 / 4(75 \%)$ \\
\hline Alcohol-free arm & $91 \%$ & $94 \%$ & $4 / 6(67 \%)$ & $4 / 6(67 \%)$ \\
\hline Overall in both arms & $90 \%$ & $94 \%$ & $6 / 10(60 \%)$ & $7 / 10(70 \%)$
\end{tabular}

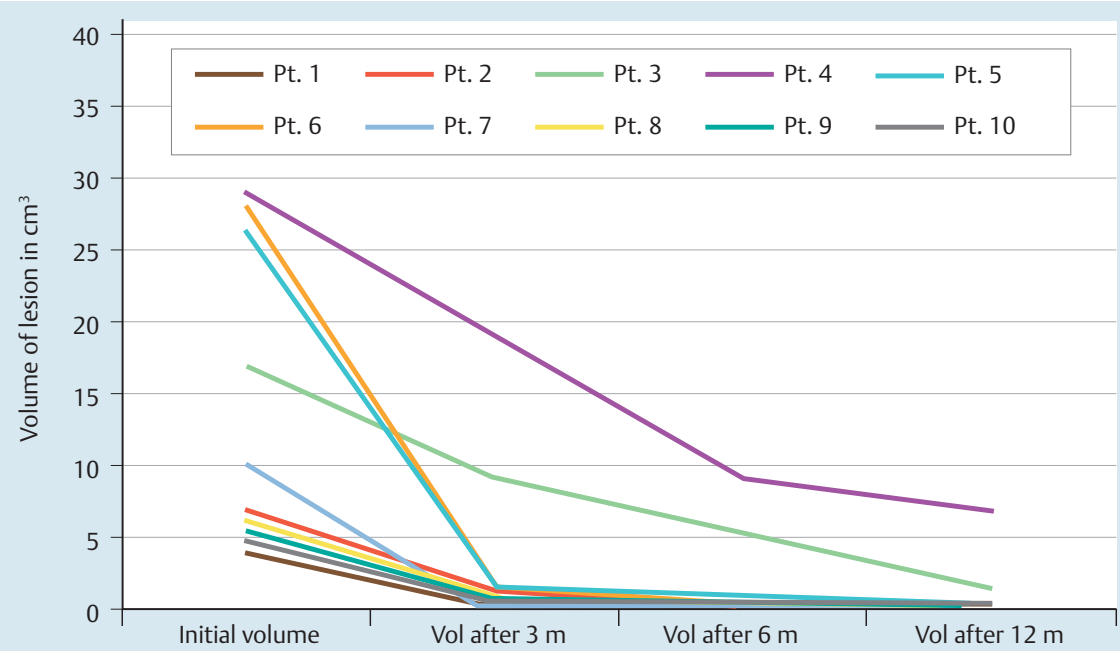

Fig. 3 Volume of cysts post-treatment

\section{Safety monitoring}

This clinical study was monitored by an independent Data Safety Monitoring Board at 6-month intervals throughout the study. This board also reviewed any interval study-related AEs in patient outcomes. AEs were documented using the standardized definitions and lexicon recommended by the FDA [16].

\section{Results \\ $\nabla$}

Between November 2011 and February 2014, 14 patients were screened for inclusion with 4 excluded and 10 patients then randomized to either the alcohol or alcohol-free arms. Reasons for exclusion included patient refusal, discovery of signs of malignancy at time of procedure, osteophyte obstruction of proximal esophagus precluding endoscopy, and discovery of excessive main duct communication with the cyst. Patients treated included 8 females and 2 males with the majority of lesions located in the body and head of the pancreas. The mean diameter of treated lesions was $30 \mathrm{~mm}$ which were predominantly unilocular. The mean value for CEA was 2,033 nanograms/mL with 7 lesions determined to be mucinous cystic neoplasms (MCNs) and 3 intra- ductal papillary mucinous neoplasms (IPMNs). Patient characteristics are detailed in $\bullet$ Table 1. At 6 months, patients randomized to the alcohol arm had an $89 \%$ average volume reduction, with a $91 \%$ reduction noted in the alcohol-free arm over the same time period ( $\odot$ Table 2). Complete ablation was achieved in $67 \%$ of patients in the alcohol-free arm at both 6 and 12 months, whereas the alcohol group recorded complete ablation rates of $50 \%$ and $75 \%$ at 6 and 12 months, respectively. One patient in the alcohol arm developed acute pancreatitis (20\%) after ablation of a $20-\mathrm{mm}$ tail cyst requiring a 36 -hour hospital stay, with no AEs in the alcohol-free arm. Also notable was that the full 1 year of surveillance was required to capture complete cyst ablation in several cases ( $\bullet$ Fig. $\mathbf{3}$ ).

\section{Discussion \\ $\nabla$}

Pancreatic cystic neoplasms are being increasingly discovered due to ever increasing use of cross-sectional imaging nationwide with an overall prevalence of $2 \%$ of all adults $[1,2]$. While many pancreatic cysts carry little to no malignant potential, the majority are neoplastic and include MCNs and IPMNs, which can carry 
a high potential for progression into pancreatic cancer. The natural history of these mucinous pancreatic cysts is not entirely clear, but the overall risk of progressing to invasive cancer is felt to be approximately $25 \%$ [1]. However, the range of this risk is wide, from negligible to quite high, and generally felt to be linked to the number of high-risk features a lesion possesses as per 2012 consensus criteria, and with MCNs having an overall higher risk of progression to malignancy [14].

Safe and effective EUS-guided ablation of pancreatic cystic lesions would lead to a significant advancement in the minimally invasive paradigm in this area of clinical pancreatology and represents an important opportunity to prevent a proportion of pancreatic cancer in selected patients. Previous trials have shown the use of alcohol in combination with paclitaxel to be relatively safe with an efficacy rate of $60 \%$ to $79 \%$, and more recent clinical trials have demonstrated the long-term durability of pancreatic cyst ablation and that the majority of patients who undergo sufficient ablation will have elimination of baseline KRAS mutations $[6,9]$. However, to date, almost all AEs have been felt to be secondary to the extravasation of alcohol into the surrounding pancreatic parenchyma, leaving some critics to question the risk profile of this procedure and limiting its more widespread clinical application $[7,8]$.

In this paper, we report the results of a prospective, randomized, double-blinded, clinical pilot study comparing an alcohol-free chemoablation approach with conventional alcohol lavage with both groups then treated with a chemotherapeutic cocktail specifically tailored to pancreatic neoplasia. The results of this pilot study demonstrate the safety and feasibility of this approach. Furthermore, this study suggests a significant discovery, namely, that ablation rates were similar for the alcohol lavage group and the alcohol-free arm (75\% vs. $67 \%$ at 12 months post-treatment). The lack of difference between alcohol and alcohol-free arms illustrates an important preliminary finding that alcohol may not be required for effective cyst ablation when a chemoablation cocktail specifically tailored for pancreatic neoplasia is used. This is significant because previous investigators have suggested that alcohol was responsible for almost all AEs (principally pancreatitis). In this study, the only AE, again pancreatitis, was noted in the alcohol arm, and while no definitive conclusions can be reached with this small sample size, this observation favors alcohol as the lone suspect as was noted in previous trials. The prospect of eliminating alcohol from pancreatic cystic lesion ablation is valid and may represent a significant step forward by improving the safety profile of this technique. A larger, randomized clinical trial to demonstrate these discoveries with statistical certainty is warranted and is currently underway with a minimum study population of 46 patients to attain adequate power.
Competing interests: Abraham Mathew: Consultant for Boston Scientific since 2013. Matthew T. Moyer: Consultant for Boston Scientific since 2012.

\section{Institutions}

${ }^{1}$ Penn State Hershey Medical Center - Gastroenterology and Hepatology, Hershey, Pennsylvania, United States

2 Penn State Hershey Medical Center - Surgical Oncology, Hershey, Pennsylvania, United States

${ }^{3}$ Penn State Hershey Medical Center - Hematology and Oncology, Hershey, Pennsylvania, United States

${ }^{4}$ Penn State Hershey Medical Center - General Internal Medicine, Hershey, Pennsylvania, United States

\section{References}

1 Ryan DP, Hong TS, Bardeesy N. Pancreatic adenocarcinoma. N Engl J Med 2014; 371: 1039-1049

2 de Jong K, Nio CY, Hermans IJ et al. High prevalence of pancreatic cysts detected by screening magnetic resonance imaging examinations. Clin Gastroenterol Hepatol 2010; 8: 806-811

3 Kiely JM, Nakeeb A, Komorowski RA et al. Cystic pancreatic neoplasms: enucleate or resect? J Gastrointest Surg 2003; 7: 890-897

4 Allen PJ, D'Angelica M, Gonen $M$ et al. A selective approach to the resection of cystic lesions of the pancreas: results from 539 consecutive patients. Ann Surg 2006; 244: 572-582

5 Goh BK, Tan YM, Cheow PC et al. Cystic lesions of the pancreas: an appraisal of an aggressive resectional policy adopted at a single institution during 15 years. Am J Surg 2006; 192: 148-154

6 Oh HC, Brugge WR. EUS-guided pancreatic cyst ablation: a critical review (with video). Gastrointest Endosc 2013; 77: 526-533

7 DeWitt J, McGreevy K, Schmidt CM et al. EUS-guided ethanol versus saline solution lavage for pancreatic cysts: a randomized, double-blind study. Gastrointest Endosc 2009; 70: 710-723

8 Oh HC, Seo DW, Lee TY et al. New treatment for cystic tumors of the pancreas: EUS-guided ethanol lavage with paclitaxel injection. Gastrointest Endosc 2008; 67: 636-642

9 DeWitt JM, Al-Haddad M, Sherman S et al. Alterations in cyst fluid genetics following endoscopic ultrasound-guided pancreatic cyst ablation with ethanol and paclitaxel. Endoscopy 2014; 46: 457-464

10 Gardner T. Best of ACG 2012. Gastroenterology \& Endoscopy News December 2012: 20-21

11 Venook AP, Egorin MJ, Rosner GL et al. Phase I and pharmacokinetic trial of gemcitabine in patients with hepatic or renal dysfunction: Cancer and Leukemia Group B 9565. J Clin Oncol 2000; 18: 2780-2787

12 Kiani A, Kohne CH, Franz T et al. Pharmacokinetics of gemcitabine in a patient with end-stage renal disease: effective clearance of its main metabolite by standard hemodialysis treatment. Cancer Chemother Pharmacol 2003; 51: $266-270$

13 Von Hoff DD, Ervin T, Arena FP et al. Increased survival in pancreatic cancer with nab-paclitaxel plus gemcitabine. N Engl J Med 2013; 369: $1691-1703$

14 Tanaka M, Fernandez-del Castillo C, Adsay Vet al. International consensus guidelines 2012 for the management of IPMN and MCN of the pancreas. Pancreatology 2012; 12: $183-197$

15 Gan SI, Thompson CC, Lauwers GY et al. Ethanol lavage of pancreatic cystic lesions: initial pilot study. Gastrointest Endosc 2005; 61: 746 752

16 Guidance for Clinical Investigators, Sponsors, and IRBs: Adverse Event Reporting to IRB's-Improving Human Subject Protection. 012009 